\title{
DISCUSSION.
}

\section{THE GALVANIC PHENOMENON.}

In a note published in The Psychological Bulletin, May ${ }_{5}$, I9Io, Mr. Dunlap passes a severe criticism on all psycho-galvanic work done until now, and especially the work done by Dr. Nelson and myself. Mr. Dunlap's objections, however, are based on several misconceptions.

First, he is wrong in thinking that we brush aside the work done by Waller, Einthoven and others. On the contrary our work is in full accord with that of the above investigators. Their work is in accord with our conclusions based on a long series of experiments that the galvanic deflections of the 'galvanic reflex' are due to an E.M.F. which is muscular in origin, since the heart is essentially a muscle its histological structure being intermediate between striated and unstriated muscle.

Second, the zero obtained by us by means of the pure platinum hypodermic electrodes is an absolute zero. There is no difference in the reading on opening or closing the circuit. This zero reading remained steady for hours. This excludes polarization of the electrodes by the tissue-fluids. The zero-reading is steady until the animal is stimulated.

Thirdly, as to the potential of different points of the body on which Mr. Dunlap lays so much stress, I can say that in all our experiments we found that it made no difference whatever as to where the hypodermic platinum electrodes were inserted. Mr. Dunlap's 'potentials' are entirely hypothetical as far as the hypodermic electrodes are concerned. We inserted platinum needle-electrodes in different parts of the body, in the same limb or in different limbs, or but a few inches apart and found no deflections whatever. This too is fully in accord with all the work done in physiology, namely that there is no current of rest.

Fourth, the platinum electrodes pick up an E.M.F. when it exists in the living or non-living animal tissues. Mr. Dunlap utterly misconceives the strength of our experiments on secretion of the shoulder and arm-pit. The E.M.F. existing in the secretion of the shoulder and arm-pit are not due to the physiological processes of secretion, but to the chemical decomposition of the products of the sudorific glands. 
The shoulder gives almost no sweat, while the arm-pit is rich in secretory glands. The E.M.F. is generated by the decomposition-products of the sweat itself. This can be demonstrated by any other nonpolarizable electrodes.

Mr. Dunlap writes "The record of the experiment in which the electrodes were moved in the tissue is not convincing. Apparently the movements were made before the galvanometer deflections had become zero." This is incorrect. The zero reading in all such experiments is absolutely steady as is clearly stated in the paper and distinctly shown in the curves.

Mr. Dunlap thinks that the galvanic reflex has not really been established. About three years ago when Dr. Kalmus and myself started on this work we had the same scepticism and objections as $\mathrm{Mr}$. Dunlap advances now. After having carried out a long series of experiments at the Physical Laboratory of the Mass. Institute of Technology, Dr. Kalmus and myself demonstrated beyond a shadow of doubt that the galvanic reflex is a fact. The present work with Dr. Nelson corroborates our former work and proves incontestably that the galvanic reflex is not due to any artefacts or to polarization, or to the action of bodily tissues, but that it is due to an electromotive force generated by muscular activity under the influence of emotional and affective states.

Mr. Dunlap says: "The topography of the potential differences has not even excited their curiosity. Sidis for example does not show that the two electrodes when placed an inch apart would not give the same deflection as when in opposite limbs." The place of insertion of the electrodes makes no difference whatever in the galvanometric deflections. The place of insertion shows no difference of potential.

Instead of speculating and advancing hypotheses about imaginary bodily potentials, Mr. Dunlap or any other doubter is invited to come to our laboratory and witness the facts. In case physiologists and psychologists or psychopathologists are not regarded as authoritative, physicists of the Physical Laboratory of the Mass. Institute of Technology will be glad to demonstrate the truth of the galvanic phenomenon and the results obtained in our investigations.

SIDIS PSYCHOTHERAPEOTIC INSTITUTE,

Boris Sidis.

PorTsModTH, N. H. 